\title{
Toward accurate volumetry of brain aneurysms: Combination of an algorithm for automatic thresholding with a 3D eraser tool
}

\author{
Vincent Costalat ${ }^{\mathrm{a}}$, Igor Lima Maldonado ${ }^{\mathrm{a}, *}$, Olivier Strauss ${ }^{\mathrm{b}}$, Alain Bonafé ${ }^{\mathrm{a}}$

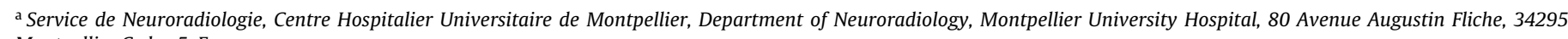 \\ Montpellier Cedex 5, France

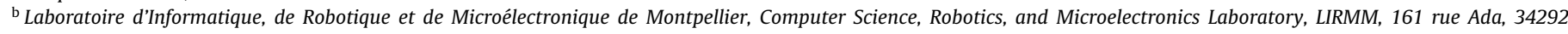 \\ Montpellier Cedex 5, France
}

\section{A R T I C L E I N F O}

\section{Article history:}

Received 4 March 2011

Received in revised form 31 March 2011

Accepted 4 April 2011

\section{Keywords:}

Aneurysm

Volume

Angiography

Digital subtraction

\begin{abstract}
A B S T R A C T
The present study describes a new approach for aneurysm volume quantification on three-dimensional angiograms, which focuses on solving three common technical problems: the variability associated with the use of manual thresholds, the irregular morphology of some aneurysms, and the imprecision of the limits between the parent artery and the aneurysm sac. The method consists of combining an algorithm for automatic threshold determination with a spherical eraser tool that allows the user to separate the image of the aneurysm from the parent artery. The accuracy of volumetry after automatic thresholding was verified with an in vitro experiment in which 57 measurements were performed using four artificial aneurysms of known volume. The reliability of the method was compared to that obtained with the technique of ellipsoid approximation in a clinical setting of 15 real angiograms and 150 measurements performed by five different users. The mean error in the measurement of the artificial aneurysms was $7.23 \%$. The reliability of the new approach was significantly higher than that of the ellipsoid approximation. Limits of agreement between two measurements were determined with Bland-Altman plots and ranged from -14 to $13 \%$ for complex and from -10.8 to $11.03 \%$ for simpleshaped sacs. The reproducibility was lower ( $>20 \%$ of variation) for small aneurysms $\left(<70 \mathrm{~mm}^{3}\right)$ and for those presenting a very wide neck (dome-to-neck ratio $<1$ ). The method is potentially useful in the clinical practice, since it provides relatively precise, reproducible, volume quantification. A safety coiling volume can be established in order to perform sufficient but not excessive filling of the aneurysm pouch.
\end{abstract}

(c) 2011 Elsevier B.V. All rights reserved.

\section{Introduction}

Aneurysm volume quantification (AVQ) is an important element of the radiological examination that precedes the endovascular treatment of cerebral aneurysms. It is an important issue in choosing the type of coil to use (Slob et al., 2005). Moreover, the Volume Embolization Ratio (VER) has been advocated as one of the best predictive factors for long-term treatment success (Cloft and Kallmes, 2004; Kawanabe et al., 2001; Raymond et al., 2003; Sluzewski et al., 2004; Tamatani et al., 2002; Vallee et al., 2005; Wakhloo et al., 2007).

Manufacturers indicate coil volume, but AVQ is still a challenging issue for interventional neuroradiologists. Most teams estimate volume by using measurements performed on two-dimensional angiograms, and mathematical ellipsoid approximation.

\footnotetext{
* Corresponding author. Tel.: +33 46733 0286; fax: +33 467337263 .

E-mail address: imaldonado@terra.com.br (I.L. Maldonado).
}

Recent improvements in the post-treatment of angiogram images have allowed three-dimensional (3D) reconstruction of the vascular tree. However, volume measurements based on the threedimensional space still require the resolution of three major technical problems (Abe et al., 2002; Anxionnat et al., 1998; Bescos et al., 2005; Carsin et al., 1997): the variability associated to the use of operator-dependent thresholds, the irregular morphology of some aneurysms, and the imprecision of the limits between the parent artery and the aneurysm sac. The latter is a major problem in case of wide-neck.

In the present study, we describe and test a new technique for aneurysm volume quantification with 3D angiograms. It consists of a combination of an algorithm for automatic determination of the optimal threshold with a three-dimensional eraser tool that allows the user to separate the aneurysm from the parent artery. The accuracy of the method was verified by an in vitro experiment with artificial silicon aneurysms of known volume. Then, a second experiment was carried out using real angiograms, in which the precision of such approach 


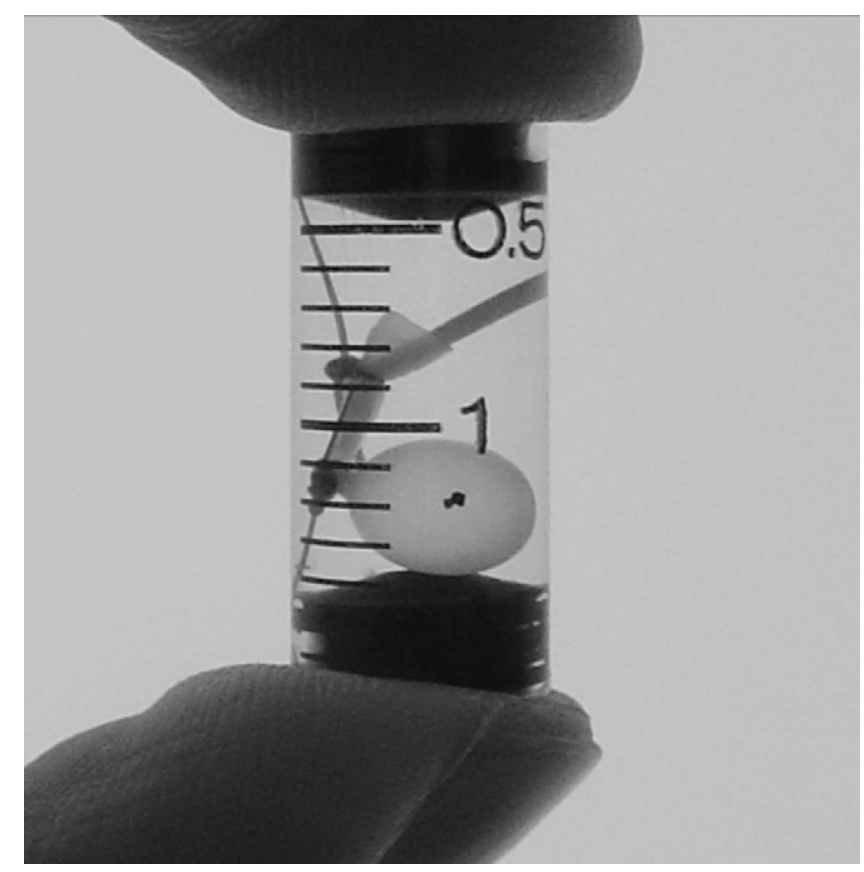

Fig. 1. An example of artificial silicone aneurysm filled with a known volume of diluted contrast medium, used to assess the accuracy of volume quantification after gradient-based edge detection.

was compared to that of the method of AVQ by ellipsoid approximation.

\section{Materials and methods}

2.1. In vitro experiment. Detection of the maximum-gradient edge in three-dimensional rotational angiograms (3D-RAs) for automatic threshold determination.

\subsubsection{Phantom}

Four silicone aneurysms (Fig. 1) of known volume (100, 200, 300 and $400 \mathrm{~mm}^{3}$ ) were used. They were filled with a solution of an iodine-based contrast medium ( $80 \%$ of Iomeron- $350 \mathrm{mg} / \mathrm{L}$ and $20 \%$ of isotonic saline) simulating the dilution obtained in the carotid circulation during a 3D-RA. The ideal concentration was calculated based on the values of a usual injection of iodinated contrast medium $(8 \mathrm{ml} / \mathrm{s})$ and the average carotid blood flow $(600 \mathrm{ml} / \mathrm{min})$. An average density of $2700 \pm 100$ Hounsfield Units (HU) was obtained, which corresponds to the values that are commonly observed in 3D-RA native images of intracranial aneurysms.

The artificial aneurysms were fixed inside a cadaver skull full with contrast medium (Iomeron- $350 \mathrm{mg} / \mathrm{L}$ ) diluted to $5 \mathrm{ml} / \mathrm{L}$ with isotonic saline. With that concentration, a mean attenuation density of $40 \mathrm{HU}$ was obtained, as commonly observed in the human brain (Bahner et al., 2005).

The whole model was placed inside a plastic recipient full with a $6 \mathrm{ml} / \mathrm{L}$ solution of contrast medium (with a density of $65 \mathrm{HU}$ ) in order to simulate the soft tissues of the extracranial space. The choice of this value of density resulted from the average of density measurements of the extracranial soft tissues in 15 healthy individuals, at six different sites.

The silicone aneurysms were placed in three different locations of the carotid circulation that are common sites of occurrence of brain aneurysms: anterior communicating, basilar and middle cerebral artery. Nineteen different 3D-RAs were performed as the aneurysms were switched from one location to another between consecutive angiograms.

\subsubsection{Threshold determination}

Before any 3D analysis, the angiographic dataset must be pretreated in order to categorize voxels into two groups: blood vessels and background.

In standard 3D analysis, this segmentation is usually achieved by manual thresholding by the practitioner. However, this may induce significant variation in the AVQ among users. Underestimation of the threshold will result in overestimation of volume, and vice versa. As a consequence, an automatic analysis for the determination of the optimal threshold for volume quantification would be advantageous.

Automatic thresholding has been a challenging point in image processing. Edge detection research has led to a reference method based on the analysis of the gradient values. However, thresholding of angiographic images is the only method that leads to appropriate $3 \mathrm{D}$ reconstruction of the vascular tree.

In this study, we use an algorithm that searches for the threshold value that provides the binary segmentation of the image with maximal agreement with the gradient-based edge.

This threshold assignment is performed in three steps. The first step consists of a gradient-based detection of the voxels belonging to 3D edges of blood vessels (and aneurysm) in the 3D image. To achieve this goal, we first compute the 3D continuous gradient vector associated with each voxel. This computation is performed by convolving the image with a 3D derivative kernel (Shen and Castan, 1992). We then locate the voxels that maximize the 3D gradient magnitude in the gradient direction, which is a property of object boundaries. This detection leads to a binary image, composed of two groups of voxels: those belonging and those not belonging to edges. The second step consists in thresholding the 3D image with different values. Then, to each one, we associate a binary image composed of two groups of voxels: those located inside the blood vessels (and the aneurysm) if the threshold is correct, and those that are not. The third step consists of finding the threshold that generates the binary image in which the object contour is closest to the binary gradient-based edge image. This search is achieved by computing the Hausdorff distance between the continuous gradient-based edge image generated in the first step and the morphological gradient-based image associated to each binary image generated in the second step (Fig. 2). The filter used to derivate the gray level image is the Shen-Castan filter whose expression in the continuous domain is

$f(x, y, z)=C_{\alpha} e^{-\alpha(|x|+|y|+|z|)}$,

where $C_{\alpha}$ is a normalization factor for the filter to have a unitary response and $\alpha$ is a spread factor. In the experiment, the value of $\alpha$ that gives the best results is $\alpha=1.0$.

Nineteen 3D-RAs of the Phantom were performed using a biplane angiographic system (Siemens, Neurostar Plus - T.O.P., Munich, Germany). Datasets were treated using a dedicated software (ELIIS, Montpellier, France), which employs the algorithm for automatic threshold determination. The surfaces of the three-dimensional objects were used to perform 57 AVQs (three aneurysms/3D-RA) (Fig. 3).

\subsection{In vivo study. Delineation of the aneurysm and parent artery using a three-dimensional (3D) eraser tool.}

\subsubsection{AVQ using the $3 D$ eraser tool}

A spherical tool was specifically developed to allow the user to erase the aneurysm sac and create the virtual image of an ideal parent artery.

Three-dimensional RAs of 15 consecutive ruptured aneurysms were performed with the same biplane angiographic system used 

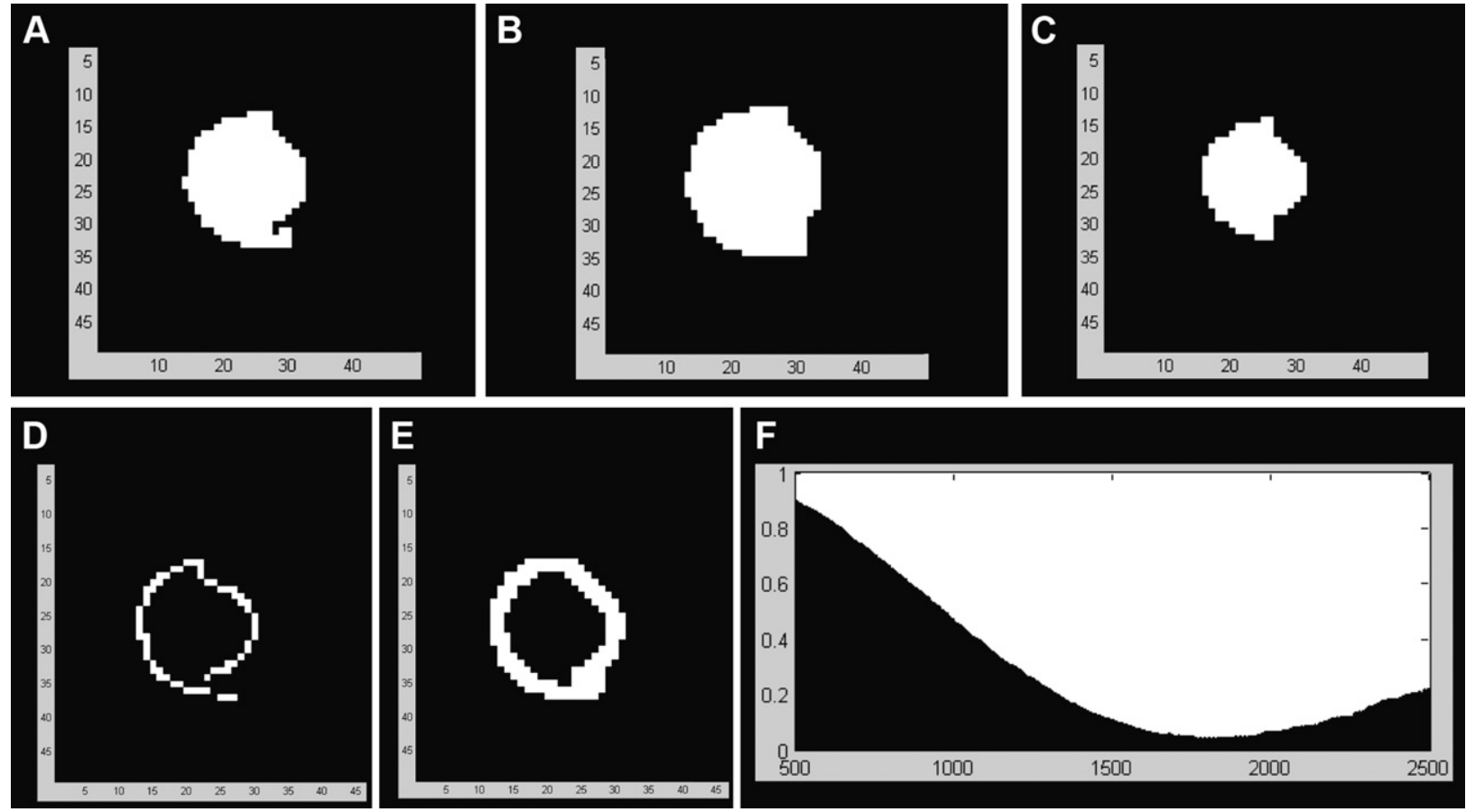

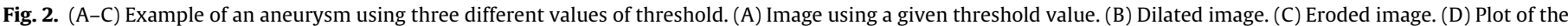

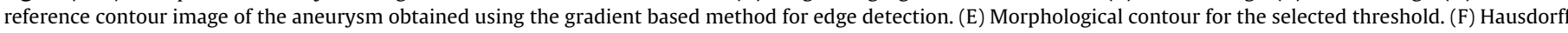

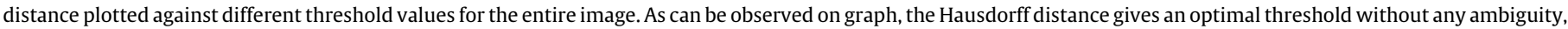
which matches with the known volume of the simulated silicone aneurysm.

in the in vitro experiment and the datasets were processed with the same automatic threshold determination software.

The objects delineated by the software were segmented with a dedicated algorithm in hexahedral volumes $\left(0.03 \mathrm{~mm}^{3}\right)$ independent from the voxel characteristics of the original images. Anytime the spherical cursor (the "3D Eraser tool") touched virtually the object (the aneurysm), the corresponding volumes were cut from the working screen and pasted in another window (Fig. 4). The diameter of the eraser tool could be adjusted to correspond to the level of accuracy desired by the operator. At the end of the procedure, the artery and the aneurysm sac were separately reconstructed. Then, the volume of the aneurysm was obtained, and the procedure stored in a file.

\subsubsection{AVQ using ellipsoid approximation}

The reconstructions of the 3D-RAs (Virtuoso, Siemens) of the same brain aneurysms were used for performing 2D measurements of the aneurysm and then a dedicated software (ELIIS, Montpellier, France) estimated its volume by means of the Ellipsoid Approximation Technique (EAT).

A multi-planar module allowed the user to choose three orthogonal diameters of each aneurysm sac. The volume $\left(V_{\text {calc }}\right)$ of each aneurysm was then calculated by means of the following formula, which gives the volume of an ellipsoid:

$V\left(\mathrm{~mm}^{3}\right)=\frac{a}{2} \times \frac{b}{2} \times \frac{c}{2} \times \frac{4}{3} \times \pi$,

where (a) denotes the largest right-left diameter, $(b)$ the largest vertical diameter and $(c)$ the largest antero-posterior diameter as measured on the 3D-RA images. The three dimensions, $(a),(b)$ and (c), are mutually orthogonal.
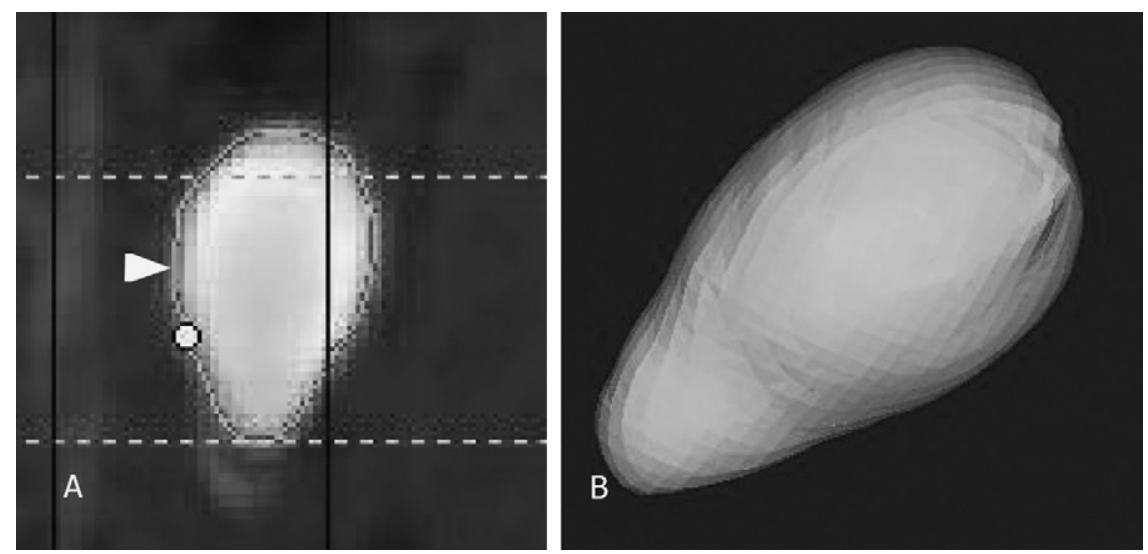

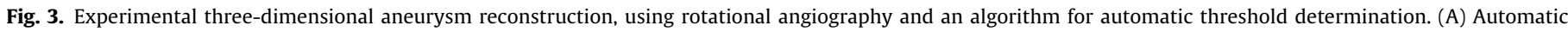

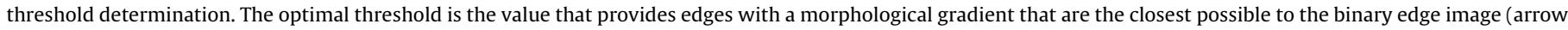
head). (B) Building of the three-dimensional aneurysm model after automatic threshold determination. No thresholding maneuver is required from the operator. 

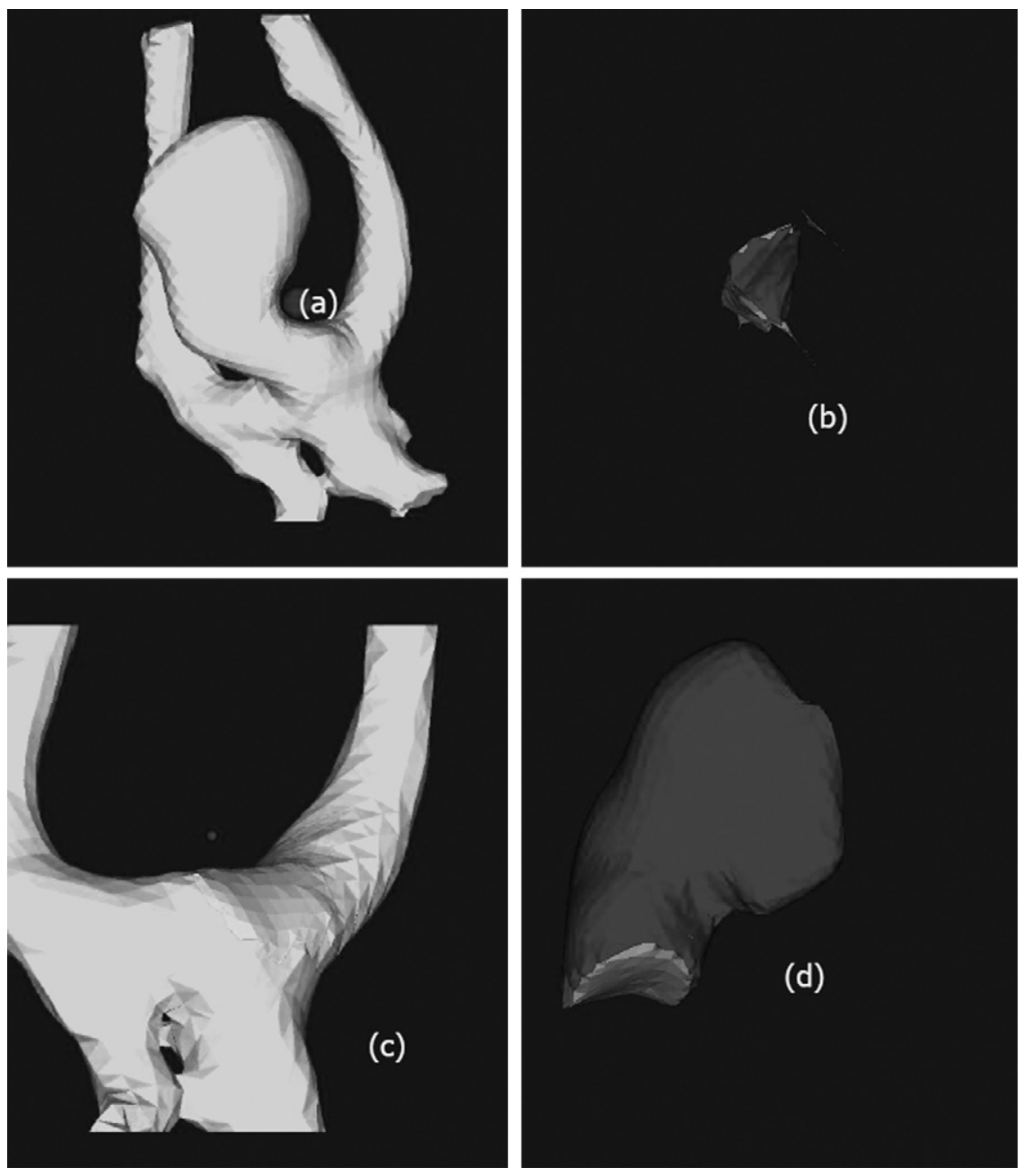

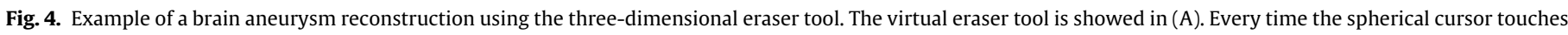

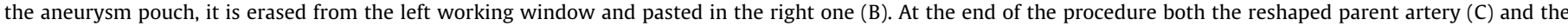
aneurysm pouch (D) are independently delineated.

\subsubsection{Volume assessment protocols}

Five different users have performed blind AVQs for each case using the 3D eraser tool: two experienced neuroradiologists, one junior radiologist, one neurosurgeon and an angiography technician. All of them had previously completed a training period in which twenty other aneurysms were studied. The goal was to obtain ideal parent artery reconstruction. Each user performed two aneurysm delineations with a two-week interval between them.

One month later, the same users performed AVQs of the same cases using the ellipsoid approximation technique. Once again, there were two measurements separated by a two-week interval. A total of 300 AVQs were accomplished: 150 per technique.

\subsubsection{Statistical analysis}

SAS Statistics v9 for Windows (SAS Institute, Cary, NC) was used for statistical analysis. The percent error among the three different anatomical locations was compared using the Kruskal-Wallis test for non-Gaussian distributions. When the difference was significant, couples of groups were treated with the Bonferroni's correction. The agreement between two different measurements was assessed with Bland-Altman plots, using a limit of acceptability of 15\% (Bland and Altman, 1986). Comparisons between two AVQ techniques were performed with a paired $t$-test. The significance threshold was 0.05 .

\section{Results}

3.1. In vitro experiment. Detection of the maximum-gradient edge in three-dimensional rotation angiograms (3D-RAs) for automatic threshold determination.

For a beam energy of $90 \mathrm{kV}$, the mean optimal threshold was $1580 \mathrm{HU}$ (range 1262-1637 HU). The average percent error of AVQs using automatic detection of the maximum gradient edge was $7.23 \pm 3.84 \%$ (mean $\pm S D$, range $0.5-13 \%$ ). The average error in volume quantification per aneurysm ranged from $3 \%$ to $11.8 \%$. A significant lower level of error was observed for aneurysms placed in the MCA in comparison to those of the ACA or BA (Table 1).

Table 1

Average percent error of AVQs by anatomical location, observed during an in vitro experiment using silicon aneurysms of known volume and automatic determination of the threshold in 3D rotational angiography images.

\begin{tabular}{lcl}
\hline Location & Average error & $p$ \\
\hline MCA & $5.2 \%$ & 0.319 \\
ACA & $10.7 \%$ & $<.0001^{\text {a }}$ \\
BA & $9.04 \%$ & $<.0001^{\text {b }}$
\end{tabular}

BA, basilar artery; MCA, middle cerebral artery; ACA, anterior communicating artery. Kruskal-Wallis test for non-Gaussian distributions.

a Comparison between MCA and ACA aneurysms.

b Comparison between MCA and BA aneurysms. 


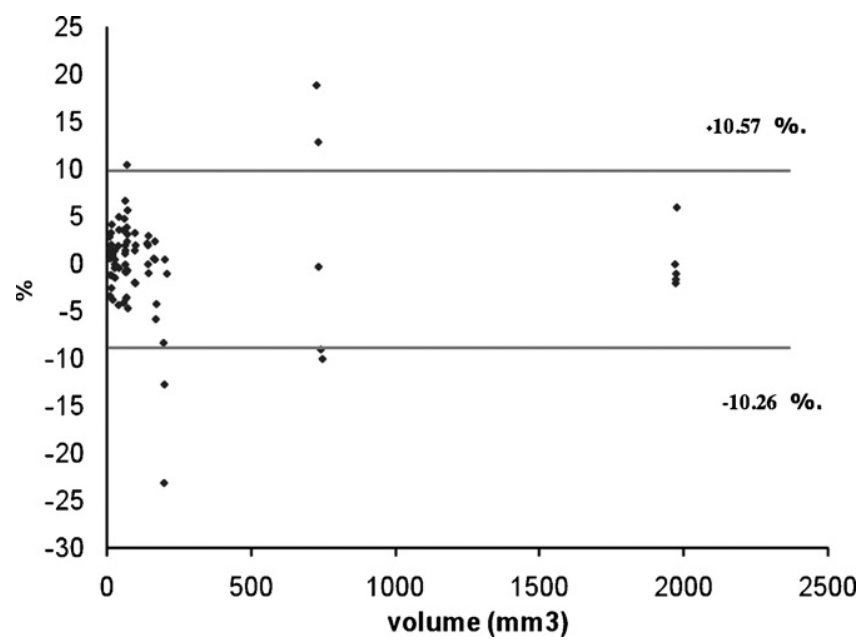

Fig. 5. Bland-Altman plot showing the degree of agreement between two AVQs (same user) with the 3D eraser tool. Each point represents the difference ( $y$-axis) in the AVQs of a brain aneurysm of a given average volume ( $x$-axis). The horizontal lines represent the limits of acceptability for the percent error in order to obtain a 95\% confidence interval. In the present study, a user would have a $95 \%$ probability of incurring an AVQ difference ranging from -10.26 to $+10.57 \%$.

\subsection{In vivo study. Delineation of the aneurysm and parent artery using a three-dimensional (3D) eraser tool.}

\subsubsection{Aneurysm characteristics}

Most of the ruptured aneurysms studied were located in the anterior circulation (13 of 15). The anatomical distribution was as following: five in the anterior communicating artery, four in the posterior communicating artery, four in the middle cerebral artery, one at the top of the basilar artery, and one in the vertebral artery. The mean aneurysm diameter was $6.01 \mathrm{~mm}$ (range $2-15 \mathrm{~mm}$ ) and the mean volume $249.02 \mathrm{~mm}^{3}$ (range $12.5-1979 \mathrm{~mm}^{3}$ ). The dometo-neck ratio ranged from 0.42 to 2.5 with a mean value of 1.2 , which characterizes a relatively wide-necked aneurysm population.

In two cases "a kissing artery" was present and visualized as an apparent fusion between the aneurysmal sac and an overlapping arterial branch. The aneurysm sac was simple-shaped, presenting a single pouch, in seven patients, and complex, multilobulated, in eight.

The mean processing time for AVQ using the three-dimensional eraser tool was 1.3 min per aneurysm. The longest processing time was $3 \mathrm{~min}$ for the largest aneurysm $\left(1973 \mathrm{~mm}^{3}\right)$.

\subsubsection{Precision of the AVQ using the three-dimensional eraser} tool

The level of reproducibility of the measurements performed with the three-dimensional eraser tool is illustrated in the Bland-Altman plot in Fig. 5. It assesses the differences between the two AVQs accomplished by the same user but separated by a two-week interval. The limits of agreement obtained here were -10.8 and $11.03 \%$. After grouping cases by morphology, the limits of agreement were -14 and $13 \%$ for complex $(n=7)$ and -10.8 and $11 \%$ for simple-shaped $(n=8)$ aneurysms, with no significant statistical difference between them.

The study of the maximum percent variation between two AVQs ( 5 trained users, 15 patients) in function of aneurysm volume is illustrated in Fig. 6, and in function of dome-to-neck ratio in Fig. 7. All major percent variations (exceeding 15\%) were concentrated in a subgroup of small $\left(<70 \mathrm{~mm}^{3}\right)$ or very wide-neck aneurysms (dome-to-neck ratio $<1$ ).

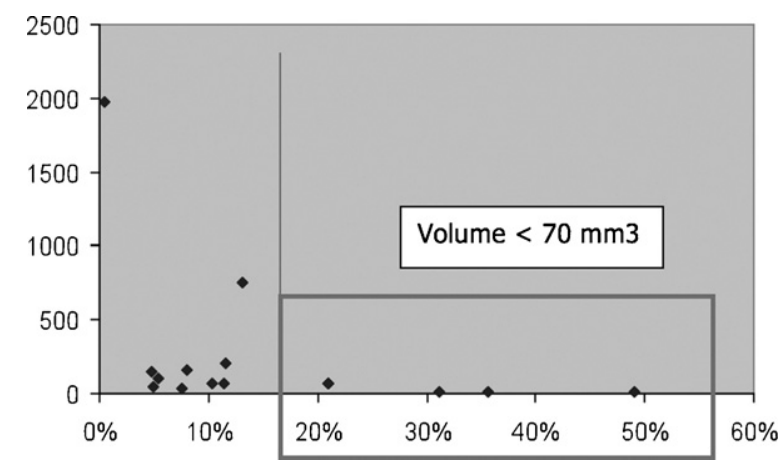

Fig. 6. Bland-Altman plot showing the maximum percent variation in the two AVQs separated by a two-week interval (five trained users, 15 patients). A maximum percent variation beyond $15 \%$ was observed in four aneurysms, all of them under $70 \mathrm{~mm}^{3}$ of volume; $x$-axis, maximal percent variation for a given aneurysm; $y$-axis, aneurysm volume in $\mathrm{mm}^{3}$.

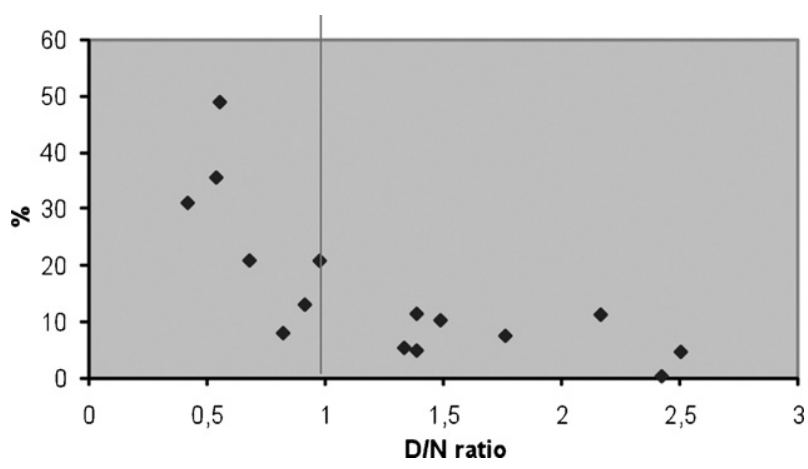

Fig. 7. Bland-Altman plot showing the maximum percent variation between two AVQs separated by a two-week interval (five trained users, 15 patients) in function of the dome-to-neck ratio. All points situated left of the vertical line (aneurysm/neck ratio < 1) represent wide-neck aneurysms. All major percent variations (exceeding $15 \%)$ were concentrated in that subgroup; $x$-axis, dome-to-neck $(\mathrm{D} / \mathrm{N})$ ratio; $y$-axis, percent variation for a given aneurysm.

The AVQs obtained with the technique of ellipsoid approximation technique presented limits of agreement of -159 and $+199 \%$. This low level of precision makes the use of Bland-Altman plot inadequate. A simple descriptive analysis was performed. Comparison with the paired $t$-test showed a very significant difference between the percent variations obtained with the two approaches, with a mean of $5 \%$ for the 3D eraser tool and of $31 \%$ for the ellipsoid approximation technique (Fig. 8).

\section{Discussion}

We presented here a new approach for AVQ using rotational digital subtraction angiography. It consists of an algorithm developed for automatic threshold determination combined with a 3D eraser tool for separating the aneurysm from the parent artery.

Despite the existence of animal aneurysm models (Bouzeghrane et al., 2010) for understanding the mechanism involved in initiation, progression, rupture, testing new endovascular devices and training interventionists, the exact volume of the aneurysm is impossible to be known, and they cannot replace an artificial aneurysm for testing volume measurement accuracy.

To make possible the study of the accuracy of volume quantification of objects delineated with such an automatic threshold, we observed the measurement errors in a series of 57 AVQs of four artificial silicon aneurysms. An average percent error of $7.23 \%$ and a maximum percent error of $13 \%$ were recorded. 


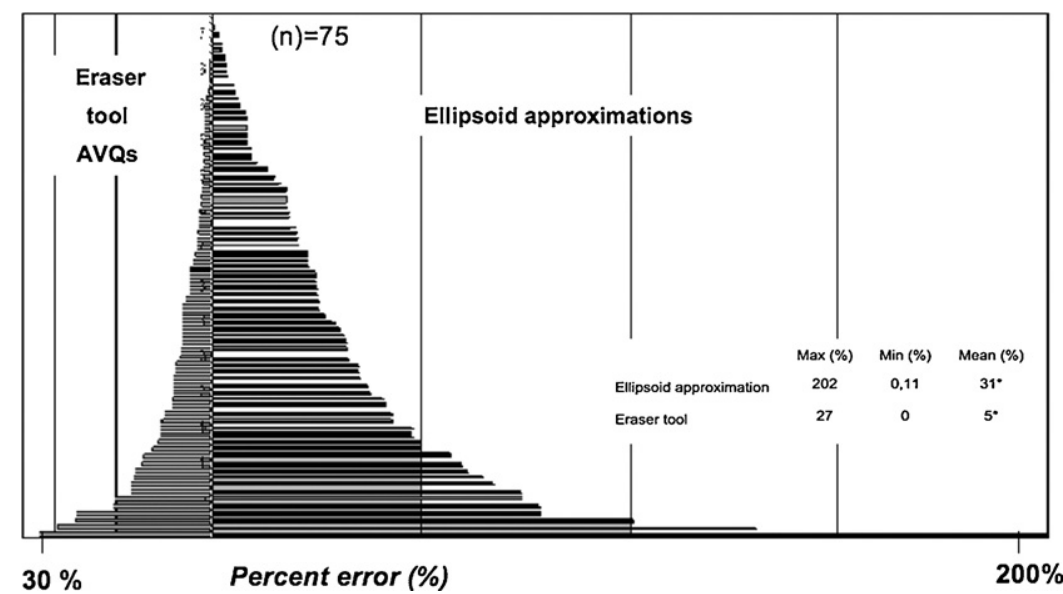

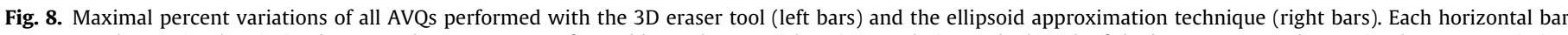

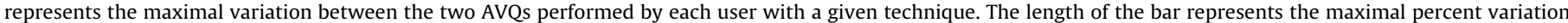
(percent error). ${ }^{*} p<0.001$. Paired $t$-test.

These figures may be acceptable for clinical purposes but are higher than those previously reported in medical literature. In 2006, Piotin et al. reported a study where an iron ball in a homogeneous gaseous environment was used for system calibration (Piotin et al., 2006). The measurement error ranged from $4 \%$ to $5.5 \%$. In the present study, aneurysms over $200 \mathrm{~mm}^{3}$ particularly tended to present more relevant measurement errors, but without any statistically significant difference.

Such an unexpected increase in the AVQ error may be related to beam-hardening artifacts and partial volume effects. Beamhardening phenomena are related to the filtering of the spectrum of X-rays by an attenuating material. With a larger object, the Xrays cross more material, which increases the beam-hardening effect. Consequently, more artifacts and measurement errors are observed. Some computerized tomography studies recommend specialized algorithms to correct beam-hardening artifacts and couple optimization tests, in order to increase the accuracy (Brown et al., 1999; van der Schaaf et al., 2006; Vannier et al., 1997).

The experiment we reported here was performed using a real skull and an artificial vascular tree that reproduced the density and anatomy of a real routine angiography. The petrous part of the temporal bone is a very dense structure, which increases beamhardening effects and may explain the distribution of the artifacts observed in our study. When an aneurysm was located in the MCA (above the petrous bone) the mean error in volume calculation was not significant (0.5\%). However, for aneurysms of the ACA or BA, very close to the skull base, or in contact to the petrous bone, the mean error increased substantially $(10 \%, p<0.001)$.

Experiments using object volume quantification without a real skull and/or a brain model would difficultly reproduce artifacts that may be responsible for major variation in volume quantification. Nevertheless, even when all precautions are taken using a realistic model, parameters that are inherent to an in vivo situation, such as pulsatility or contrast dilution variation are still problematic.

Indeed, in a real situation, as in the "in vivo" phase of our study, the inadvertent heterogeneity of the contrast medium dilution could be a potential source of imperfect aneurysm opacification and volume measurement error. This may be seen with large, irregular shaped aneurysms, with a turbulent flow in the dome. Giant aneurysms are still a challenging issue for 3D RA-based volumetry and may motivate adaptation of the contrast medium injection protocol in a case-by-case basis in order to obtain optimal opacification.

Variations in the beam X-ray energy may also potentially induce changes in the aneurysm sac depiction. As recently verified by
Murakami et al. (2010), in a series of cerebral computerized tomography, large variations of the X-ray beam energy (both reduction or augmentation) induce degradation in contrast and diagnostic imaging quality regarding the depiction of the simulated cerebral vascular lesions. In comparison to computerized tomography, it is important to remember that in cerebral angiography, X-ray beam energy do not present significant variation using the same angiography suite once it is correctly calibrated. In this last case, what is more variable between two acquisitions is not the $\mathrm{X}$-ray beam energy, but the peri-aneurysmal environment inherent to each patient (individual bone and soft tissue structures).

The three-dimensional gradient edge detection algorithm seeks for the voxels belonging (or intersecting) the aneurysm envelope. This is less sensitive to the global energy of the 3D image, but it is sensitive to the relative values associated with each voxel. As a consequence, this method provides a threshold value that is less sensible to peri-aneurysmal environment variations.

Ellipsoid approximation has the disadvantage of presenting substantial variability of results. It is not a reliable tool, even when assisted by multiplanar resources. In our team's experience, it may be responsible for variations as high as $31 \%$. Similar observations were made by Piotin et al. (2006).

A relevant number of aneurysms studied here were challenging, due to their small size (mean volume $249 \mathrm{~mm}^{3}$, mean diameter $6 \mathrm{~mm}$ ) or wide neck (12 of 15 aneurysms). The 3D eraser tool showed to be more precise than EAT, and then more adequate for clinical use. The fact that the image of the aneurysm can be separated from the parent artery assures adequate delineation, which is not easily obtained with automatic image treatment. Furthermore, the method keeps its precision for complex-shaped aneurysms.

This technique has two relative limitations: small aneurysms and extremely wide necks. When the aneurysm volume was less than $70 \mathrm{~mm}^{3}$, or the "dome-to-neck ratio" was under 1 , the maximum percent errors observed often exceeded 15\%. For small aneurysms (under $70 \mathrm{~mm}^{3}$ ), the variability among practitioners in delineating aneurysm boundaries was proportionally more significant, because the volume of the sac is smaller. In other words, small variations in erasing the parent area and delineating the aneurysm neck could lead to significant percent variations of the AVQ because the AVQ was small. This led to a wider range of measurement results (and larger variations in AVQ, over 15\%). In very wide neck aneurysms (dome-to-neck ration $<1$ ), the parent artery was often dysplasic and more than $50 \%$ of its cross-section belonged to the aneurysm neck. The delineation of such a neck may be very difficult, which may be at the origin of some inter-observer variation. 
Furthermore, this automatic choice could certainly lead to poor visualization of some anatomic details on the 3D images. Another threshold setting might be more appropriate for anatomic depiction for therapeutic planning purposes. As a consequence, the practitioner can manually set his preferred threshold once the volume quantification is done.

The assessment of the volume of a cerebral aneurysm is a very important issue in clinical practice. It allows avoidance of overfilling during the endovascular procedure and reduces the per-operative risks. A safety volume $\left(V_{S}\right)$ may be obtained by a deliberate underestimation of the aneurysm volume to be filled by coils. This corresponds to the AVQ deducted of the sum of all possible errors, i.e. $17.7 \%$ in the present study:

$V_{s}=V_{c}(1-\mathrm{GE})$,

or

$V_{S}=V_{C} \cdot 0.823$ (present study),

where " $V_{c}$ " is the aneurysm volume obtained by a given AVQ technique and "GE" the corresponding maximal global error. The probability of the real aneurysm volume to be higher than $V_{S}$ is maximal. Therefore, " $V_{s}$ " is the security level that may be used for therapeutic planning, and may be taken into consideration when establishing a target VER.

\section{Conclusion}

In the present study we presented a new approach for aneurysm volume quantification using $3 \mathrm{D}$ rotational angiograms, which focuses on reducing common measurement errors that are associated to inter-observer variability, neck width, or morphological complexity of the aneurysm. The combination of an algorithm for automatic detection of the optimal density threshold and a threedimensional eraser tool for delineation of the parent artery was able to provide volume quantification with acceptable accuracy, reproducibility, and a short processing time, except for small $\left(<70 \mathrm{~mm}^{3}\right)$ or extremely wide-neck aneurysms (dome-to-neck ratio $<1$ ).

Respecting the limits that are inherent to such a method, this is a potentially useful instrument in daily clinical practice, and more reliable than volume estimation by ellipsoid approximation. The maximal cumulative error of the technique is roughly $18 \%$, which allows interventionists to calculate a safety volume that can be taken into consideration when setting the target VER for coiling.

\section{References}

Abe T, Hirohata M, Tanaka N, Uchiyama Y, Kojima K, Fujimoto K, et al. Clinical benefits of rotational 3D angiography in endovascular treatment of ruptured cerebral aneurysm. AJNR Am J Neuroradiol 2002;23:686-8.
Anxionnat R, Bracard S, Macho J, Da Costa E, Vaillant R, Launay L, et al. 3D angiography. Clinical interest. First applications in interventional neuroradiology. J Neuroradiol 1998;25:251-62.

Bahner ML, Bengel A, Brix G, Zuna I, Kauczor HU, Delorme S. Improved vascular opacification in cerebral computed tomography angiography with $80 \mathrm{kVp}$. Invest Radiol 2005;40:229-34.

Bescos JO, Slob MJ, Slump CH, Sluzewski M, van Rooij WJ. Volume measurement of intracranial aneurysms from 3D rotational angiography: improvement of accuracy by gradient edge detection. AJNR Am J Neuroradiol 2005;26:2569-72.

Bland JM, Altman DG. Statistical methods for assessing agreement between two methods of clinical measurement. Lancet 1986;1:307-10.

Bouzeghrane F, Naggara O, Kallmes DF, Berenstein A, Raymond J, International Consortium of Neuroendovascular Centres. In vivo experimental intracranial aneurysm models: a systematic review. AJNR Am J Neuroradiol 2010;31:418-23.

Brown JH, Lustrin ES, Lev MH, Ogilvy CS, Taveras JM. Reduction of aneurysm clip artifacts on CT angiograms: a technical note. AJNR Am J Neuroradiol 1999;20:694-6.

Carsin M, Chabert E, Croci S, Romeas R, Scarabin JM. The role of 3-dimensional reconstructions in the angiographic evaluation of cerebral vascular malformations: $3 \mathrm{D}$ morphometry. J Neuroradiol 1997;24:137-40.

Cloft HJ, Kallmes DF. Aneurysm packing with HydroCoil Embolic System versus platinum coils: initial clinical experience. AJNR Am J Neuroradiol 2004;25:60-2.

Kawanabe Y, Sadato A, Taki W, Hashimoto N. Endovascular occlusion of intracranial aneurysms with Guglielmi detachable coils: correlation between coil packing density and coil compaction. Acta Neurochir (Wien) 2001;143:451-5.

Murakami Y, Kakeda S, Kamada K, Ohnari N, Nishimura J, Ogawa M, et al. Effect of tube voltage on image quality in 64-section multidetector 3D CT angiography: evaluation with a vascular phantom with superimposed bone skull structures. AJNR Am J Neuroradiol 2010;31:620-5.

Piotin M, Daghman B, Mounayer C, Spelle L, Moret J. Ellipsoid approximation versus 3D rotational angiography in the volumetric assessment of intracranial aneurysms. AJNR Am J Neuroradiol 2006;27:839-42.

Raymond J, Guilbert F, Weill A, Georganos SA, Juravsky L, Lambert A, et al. Long-term angiographic recurrences after selective endovascular treatment of aneurysms with detachable coils. Stroke 2003;34:1398-403.

Shen J, Castan S. An optimal linear operator for step edge detection computer vision. Graph Image Process 1992;54:112-3.

Slob MJ, van Rooij WJ, Sluzewski M. Coil thickness and packing of cerebral aneurysms: a comparative study of two types of coils. AJNR Am J Neuroradiol 2005;26:901-3.

Sluzewski M, van Rooij WJ, Slob MJ, Bescos JO, Slump CH, Wijnalda D. Relation between aneurysm volume, packing, and compaction in 145 cerebral aneurysms treated with coils. Radiology 2004;231:653-8.

Tamatani S, Ito Y, Abe H, Koike T, Takeuchi S, Tanaka R. Evaluation of the stability of aneurysms after embolization using detachable coils: correlation between stability of aneurysms and embolized volume of aneurysms. AJNR Am J Neuroradiol 2002;23:762-7.

Vallee JN, Pierot L, Mont'alverne F, Turjman F, Bonafe A, Bracard S, et al. Unruptured intracranial aneurysms treated by three-dimensional coil embolization: evaluation of the postoperative aneurysm occlusion volume. Neuroradiology 2005;47:438-45.

van der Schaaf I, van Leeuwen M, Vlassenbroek A, Velthuis B. Minimizing clip artifacts in multi CT angiography of clipped patients. AJNR Am J Neuroradiol 2006;27:60-6.

Vannier MW, Hildebolt CF, Conover G, Knapp RH, Yokoyama-Crothers N, Wang G. Three-dimensional dental imaging by spiral CT.A progress report. Oral Surg Oral Med Oral Pathol Oral Radiol Endod 1997;84:561-70.

Wakhloo AK, Gounis MJ, Sandhu JS, Akkawi N, Schenck AE, Linfante I. Complexshaped platinum coils for brain aneurysms: higher packing density, improved biomechanical stability, and midterm angiographic outcome. AJNR Am J Neuroradiol 2007;28:1395-400. 